\title{
Tempo de Fluxo Carotídeo como Preditor de Fluidorresponsividade: Revisão Narrativa
}

\author{
Carotid Flow Time as a Predictor of Fluidresponsiveness: \\ A Narrative Review
}

Correspondence addresses:

Dr. Elton Pereira de Sá Barreto Júnior

elton.sa.junior@gmail.com

Received: December 11, 2019

Revised: January 21, 2020

Accepted: January 23, 2020

Published: March 31, 2020

Data Availability Statement: All relevant data are within the paper and its Supporting Information files.

Funding: This work was the result of authors' initiative. There was no support of research or publication funds.

Competing interests: The authors have declared that no competing interests exist.

Copyright

(C) 2020 by Santa Casa

de Misericórdia da Bahia.

All rights reserved.

ISSN: 2526-5563
A monitorização hemodinâmica constitui pilar essencial no manejo perioperatório de pacientes de alto risco e/ou em cirurgias de grande porte. A reposição volêmica deve ser idealmente guiada por metas, objetivando um melhor prognóstico dos pacientes. Ao longo dos anos, diferentes métodos para avaliação do débito cardíaco foram desenvolvidos a partir da introdução do catéter de Swan Ganz, que utiliza o princípio da termodiluição. Sistemas que analisam as curvas de pressão arterial e métodos ultrassonográficos ganharam espaço nos últimos anos. Nesse contexto, torna-se importante citar o uso do tempo de fluxo carotídeo corrigido, ainda com pequena evidência de validação na literatura, mas descrito como uma medida acurada de fluidorresponsividade, de simples e fácil execução. Foi realizada uma revisão de estudos sobre medidas de fluxo carotídeo como preditor de fluidorresponsividade nas plataformas PubMed e UptoDate, com estudos incluídos de 2015 a 2018. As medidas citadas nos estudos foram: fluxo sanguíneo em carótida, tempo de fluxo carotídeo e variação de velocidade de pico sistólico de carótida. Diversos trabalhos foram conduzidos em diferentes cenários na tentativa de correlacionar medidas de fluxo em artéria carótida com fluidorresponsividade. Em alguns deles, provas volêmicas foram realizadas, seja com soluções cristaloides ou através de "passive leg raise", com variação significativa em medidas de fluxo na referida artéria. Em um cenário distinto, de hipovolemia aguda após trauma, o tempo de fluxo carotídeo diminuiu após a perda sanguínea. Em estudo mais recente, já considerando tal medida como acurada na predição de fluidorresponsividade, valores de cut-off foram determinados para os diferentes gêneros. Medidas de fluxo em artéria carótida representam medidas dinâmicas, não invasivas, de fluidorresponsividade, de modo que mudanças nessas medidas estão associadas a alterações no status volêmico. Contudo, estudos clínicos controlados e randomizados precisam ser realizados para confirmar a real acurácia do método.

Palavras-chave: Artéria Carótida; Fluxo Carotídeo; Fluidorresponsividade.

Hemodynamic management is an essential mainstay in the perioperative of significant surgeries and high-risk patients. Volume replacement should be guided by goals, aiming at a better prognosis for patients. Over the years, the different methods for assessing cardiac output have been developed since the introduction of the Swan Ganzcatheter, which uses the principle of thermodilution. Systems that analyze blood pressure curves and ultrasound methods have gained ground in recent years. So, the use of corrected carotid flow time is an accurate measure of fluid responsiveness, simple and easy to perform, even with little evidence in the literature. We reviewed studies on carotid flow measurements as a predictor of fluid responsiveness on the 
PubMed and UptoDate platforms from 2015 to 2018. The measures cited in the studies were: blood flow in carotid, time of carotid flow, and speed variation systolic carotid peak. Several studies conducted attempt to correlate flow measurements in the carotid artery with fluid responsivenessin in different scenarios. In some studies, volemic tests were carried out, either with crystalloid solutions or through a "passive leg raise", with significant variation in flow measures in the artery. The carotid flow time decreased after bloodloss in a different condition of acute hypovolemia after trauma. A recent study determined cut-off values for defferent genders, already considering this measure as accurate in predicting fluid responsiveness. Flow measurements in the carotid artery represent dynamic, non-invasive, fluid responsiveness measures, so that changes in these measures are associated with changes in fluid status. However, controlled and randomized trials need to be carried out to confirm the real accuracy of the method.

Keywords: Carotid Artery; Carotid Flow; Fluidresponsiveness.

\section{Introdução}

A reposição volêmica adequada é um dos objetivos do manejo perioperatório e faz parte dos pilares do protocolo ERAS (Enhanced Recovery After Surgery), permitindo, dentre outras coisas, uma recuperação fast track na Unidade de Terapia Intensiva. A administração inadequada de fluidos está associada a um pior desfecho clínico do paciente. O excesso de líquido leva a consequências catastróficas, como deiscência de alças, fístulas, translocação bacteriana e lesões pulmonares, ao passo que a reposição insuficiente resulta em hipoperfusão tecidual e isquemia orgânica. ${ }^{1}$

O catéter de artéria pulmonar foi introduzido na prática clínica na década de 70 pelos seus idealizadores Jeremy Swan e William Ganz. Seu uso foi ampliado para monitorização hemodinâmica do paciente grave e como guia terapêutico. Por muitos anos, foi considerado o padrão-ouro para medida do débito cardíaco, através do método de termodiluição pulmonar. Contudo, em razão dos riscos inerentes à passagem e manutenção do catéter, novos métodos foram desenvolvidos e aplicados à prática clínica. ${ }^{1}$

Durante os últimos 10 anos, técnicas minimamente ou não invasivas foram incorporadas à prática clínica para avaliação e cuidado do paciente crítico. Monitores mais modernos, com algoritmos próprios, ganharam espaço em detrimento de métodos mais antigos de monitorização, que oferecem risco devido ao caráter invasivo.
Sabemos hoje que medidas dinâmicas de fluidorresponsividade, baseadas em interação coração-pulmão, como variação da pressão de pulso (delta $\mathrm{PP}$ ), variação de volume sistólico (VVS) e índice de variabilidade pletismográfica, têm sido usadas como guia de reposição volêmica em detrimento de medidas estáticas, como pressão de oclusão de artéria pulmonar ou pressão venosa central, que demonstraram pequena acurácia em predizer reposta ao desafio volêmico. ${ }^{2}$

Nos últimos anos, os métodos ultrassonográficos de status volêmico estão em evidência em razão da sua confiabilidade e do seu caráter minimamente invasivo. Dentre as modalidades ultrassonográficas, medidas de fluxo em artéria carótida ganham crescente interesse como preditor de fluidorresponsividade, com resultados promissores. ${ }^{3}$

Pouco foi descrito na literatura a respeito dessa medida, mas estudos prévios demonstraram que mudanças no tempo de fluxo carotídeo correlacionam-se com alterações no status volêmico. Sendo assim, tal ferramenta pode representar uma medida não invasiva de fluidorresponsividade, útil e de fácil obtenção, mesmo em mãos inexperientes.

Diante do exposto, o objetivo desse estudo é fazer uma revisão narrativa de estudos sobre medidas de fluxo em artéria carótida, determinadas por ultrassonografia com Doppler, como preditoras de fluidorresponsividade.

\section{Materiais e Métodos}

Trata-se de uma revisão narrativa, realizada 
nas plataformas PubMed e UptoDate, sobre medidas de fluxo em artéria carótida como preditoras de fluidorresponsividade. Foram incluídos estudos entre 2015 a 2018 . Utilizaramse as seguintes palavras-chave, na língua inglesa: blood flow velocity, carotid flow, carotid Doppler, systolic carotid flow, cardiac output, volume responsiveness, Doppler ultrasound, fluid therapy, carotid artery.

Dentre os estudos encontrados, foram descritas as seguintes medidas em artéria carótida: fluxo sanguíneo em carótida, tempo de fluxo carotídeo e variação de velocidade de pico sistólico em carótida.

Tais estudos foram revisados, selecionados e agrupados de acordo com a ordem cronológica de publicação e a real abordagem sobre o tema.

\section{Resultados}

Medidas de fluxo em artéria carótida podem ser utilizadas, dentro do atual conceito de monitorização hemodinâmica minimamente invasiva, como preditores de fluidorresponsividade. Estudos recentes demonstram tal aplicação em diferentes cenários.

No estudo de Mackenzie e colegas, ${ }^{4}$ o fluxo sanguíneo em artéria carótida foi medido antes e após perda sanguínea. Tal fluxo apresentou redução no cenário de hipovolemia aguda e, após a realização de "passive leg raise", os valores de fluxo foram restaurados a valores anteriores, demonstrando que tal medida pode melhorar o cuidado do paciente no departamento de emergência.

A aplicabilidade do tempo de fluxo carotídeo foi avaliado por Shokoohi e colegas. ${ }^{5}$ Em seu estudo, considera tal medida como parte da avaliação ultrassonográfica focada (FoCUS), sendo capaz de prover medida não invasiva e de acurácia confiável de status volêmico e de resposta a provas volêmicas em pacientes com jejum prolongado e submetidos à manobra de "passive leg raise". Uma mudança maior ou igual a 5\% no tempo de fluxo carotídeo relaciona-se à resposta volêmica positiva.

Em um cenário de pacientes criticamente enfermos em UTI, Jalil e colegas, ${ }^{6}$ avaliaram a aplicação do tempo de fluxo carotídeo na predição de fluidorresponsividade. Utilizou-se o Vigileo/Flotrac como medida de débito cardíaco e, em caso de aumento do índice cardíaco maior que $15 \%$, o paciente era considerado como fluidorresponsivo. Nesses pacientes, o tempo de fluxo carotídeo teve bom desempenho, com variações significativas após manobra de "passive leg raise", considerado bom instrumento preditor de fluidorresponsividade.

Já considerando a avaliação tempo de fluxo carotídeo corrigido como medida de status volêmico, Hossein-Nejad e colegas ${ }^{7}$ objetivaram determinar valores de referência de tal medida e valores de cutoff na avaliação do fluxo carotídeo predizendo responsividade a fluidos.

Em outro estudo publicado por Sidor e colegas, ${ }^{8}$ foram avaliadas medidas em artéria carótida (fluxo sistólico, fluxo total e tempo de fluxo corrigido) em 20 voluntários saudáveis, no estado basal e após manobra de "passive leg raise". Todas essas medidas correlacionaram-se a fluidorresponsividade após a manobra, com variações proporcionais ao índice cardíaco, confirmando-as como de valor para avaliação do status hemodinâmico.

Utilizando o ecocardiograma transtorácico como medidor de débito cardíaco, Kim e colegas $^{9}$ avaliaram pacientes em ventilação espontânea antes da indução anestésica para neurocirurgia. As medidas de fluxo em carótida foram realizadas antes e após prova volêmica com $6 \mathrm{~mL} / \mathrm{kg}$ de solução coloide e a fluidorresponsividade foi definida como o aumento de índice de volume sistólico acima de $15 \%$. Tais medidas foram consideradas acuradas em predizer fluidorresponsividade para pacientes em ventilação espontânea.

Em uma unidade de terapia intensiva (UTI), em grande centro acadêmico, Barjaktarevic 
e colegas $^{10}$ aplicaram o tempo de fluxo carotídeo em pacientes criticamente enfermos, com choque indiferenciado em fase precoce. Utilizou-se um monitor não invasivo de débito cardíaco, baseado em biorectância, e variações superiores a $10 \%$ no volume sistólico foram consideradas para os fluidorresponsivos. Nesse grupo de pacientes, o tempo de fluxo carotídeo corrigido foi medido antes e após "passive leg raise", sendo considerado um método aceitável, não invasivo e reprodutível de responsividade volêmica, dentro da avaliação ultrassonográfica point of care.

As principais limitações da presente revisão foram: ausência de estudos com um ambiente cirúrgico controlado (cenário do anestesiologista), com técnicas anestésicas padronizadas, ausência de estudos comparativos com outras medidas de fluidorresponsividade, como variação de pressão de pulso ou variação de volume sistólico e falta de estudos que utilizem o Swan Ganz, ainda considerado padrão-ouro, como medidor do débito cardíaco. ${ }^{11}$

\section{Conclusão}

As medidas de fluxo em artéria carótida podem ser consideradas como preditores de fluidorresponsividade. Representam, portanto, medidas ultrassonográficas dinâmicas de status volêmico, não invasivas, de fácil obtenção e de baixo custo. Dessa forma, tais medidas devem ganhar cada vez mais espaço no cenário atual, com o advento da monitorização minimamente invasiva, em cirurgias de grande porte. Contudo, trials randomizados e controlados devem ser conduzidos para confirmar a real acurácia do método em predizer fluidorresponsividade e otimizar o manejo volêmico quando comparado a medidas bem estabelecidas.

\section{Referências}

1. Assunção E, Fernandes HS. Monitorização hemodinâmica no paciente grave. $1^{a}$ ed. São Paulo: Editora Atheneu, 2013.

2. Suehiro K, Tanaka K, Matsuura T et al. The VigileoFloTrac $^{\mathrm{TM}}$ System: arterial waveform analysis for measuring cardiac output and predicting fluid responsiveness:aclinicalreview.JournalofCardiothoracic and Vascular Anesthesia.2014;28(5):1361-1374.

3. Barjaktarevic I, Chiem A, Cannesson M. Time to correct the flow of corrected flow time. Critical Ultrasound Journal. 2017.

4. Mackenzie DC, Khan NA, Blehar D et al. Carotid flow time changes with volume status in acute blood loss. Annals of Emergency Medicine. 2015.

5. Shokoohi H, Berry GW, Shahkolahi $M$ et al. The diagnostic utility of sonographic carotid flow time in determining volume responsiveness. Journal of Critical Care. 2016.

6. Bilal J, Patton T, Cavallazzi R et al. Comparing changes in carotid flow time and stroke volume induced by passive leg raising. The American Journal of the Medical Sciences.2017. http://dx.doi.org/10.1016/j. amjms.2017.09.006.

7. Hossein-Nejad H, Banaie M, Davarani SS et al. Assessment of corrected flow time in carotid artery via point-ofcareultrasonography: Reference values and the influential factors. Journal of Critical Care. 2017;42:46-51.

8. Sidor M, Premachandra L, Hanna B et al. Carotid flow as a surrogate for cardiac output measurement in hemodynamically stable participants. Journal of Intensive Care Medicine. 2018.

9. Kim DH, Shin S, Kim N et al. Carotid ultrasound measurements for assessing fluid responsiveness in spontaneously breathing patients: corrected flow time and respirophasicvariation in blood flow peakvelocity. British Journal of Anaesthesia. 2018;121(3):541-549.

10. Barjaktarevic I, Toppen WE, HU S et al. Ultrasound assessment of the change in carotid corrected flow time in fluidresponsiveness in undifferentiated shock. Critical Care Medicine. 2018.

11. Sotomi Y, Iwakura K, Higuchi Y et al. The impact of systemic vascular resistance on the accuracy of the FloTrac/Vigileo ${ }^{\mathrm{TM}}$ system in the perioperative period of cardiac surgery: a prospective observational comparison study. J Clin Monit Comput. 2013. DOI 10.1007/s10877-013-9481-2. 